\title{
Performance of Efficient RLS Adaptive Algorithm Used to Enhance the ECG Signal Quality in Telecardiology
}

\author{
Gowri T. \\ Dept. of ECE, GITAM \\ University, Visakhapatnam- \\ 530045, AP INDIA
}

\author{
Rajesh Kumar P. \\ Dept. of ECE, Andhra \\ University, Visakhapatnam- \\ 530003, AP INDIA
}

\begin{abstract}
When acquiring the Electrocardiogram (ECG) signal from the person, it should be preprocess before sending to the analyst for taking decision of the signal, because signal should be affected with various artifacts. For numerous applications of noise cancellation in the corrupted signals, adaptive filters play important role. The various artifacts which commonly occur in the acquisition of ECG signals are physiological and non- physiological noises, those are main supply power line interference, muscle artifact, electrode motion artifact and base line wander noises. The adaptive Least Mean Square (LMS) algorithm provides a low convergence rate, so that for fast convergence rate and reduced noise, in this paper an efficient Recursive Least Square algorithm is considered, for removing of power line noise and muscle noise. For double validation of the signal, and for high Signal to Noise Ratio (SNR), fast convergence rate, is achieved by using LMS to RLS adaptive algorithm at the cost of additional computations.
\end{abstract}

\section{Keywords}

Adaptive algorithm, RLS algorithm, SNR, Artifacts, Convergence rate.

\section{INTRODUCTION}

When acquiring bio signals from the human body several artifacts which will strongly affects the frequency resolution of the signal and also changes the amplitude of the signal [1]. The problem of interference in ECG signal is addressed by James et al. in [2]. In the biological signals the ECG signal play important role, which gives the different functionality of the heart: some of them are priori heart attach information, any effect of drugs in the heart, decreased oxygen delivery into the heart and abnormal spread of electrical impulses across the heart. The ECG signal is series of waves which occurs due to electrical activity of the body that is depolarization and re-polarization of shifting of electrolytes cells. In clinical laboratory when measuring the signal, it is affected by different noises, due to these noises the original signal characteristics changes.

The various types of noises which degrade the pure ECG signal are Power Line Interference (PLI), measuring instrument noise, electrode motion (EM) noise, respiration noise and Muscle artifact (MA) noise. When transmitting the ECG signal from remote place to diagnosis center, signal through [3] telecardiology system, for analysis of the signal there is additional channel noise and electromagnetic noise also added to the pure signal.

One of the applications in telecardiology is wireless Ambulatory [4-5] ECG system. A block diagram of a wireless ambulatory system is shown in Fig 1. In this system, ECG signal is a bioelectrical signal and is used to know the cardiac condition of an ambulatory patient. The wireless Ambulatory ECG monitoring has been used to detect arrhythmias. During the acquisition of the ECG signal using electrodes which were placed on the body, the ECG signal will undergo numerous artifacts. Sometimes using GSM modem with attached antenna also used to transmit the stored signal. The expert at the real time diagnosis center can receive the data and analyzed for correct decision on the signal and give instructions to the ambulance center for action of the patient. When transmitting signal for analysis, the channel noise to be considered and remove at the receiver side.

So better diagnosis of the patient ECG signal and getting high accuracy of signal: be achieved by reducing the various noises affected during acquisition and transmission, using different techniques. We can use adaptive and non adaptive filters to reduce the noises in signal [6-10]. The most dominating technique for removing of noises in desired signal is adaptive filters. The adaptive algorithms which are used to adjust the filter coefficients, in such way that the error in the desired signal to be reduced based on mean square or least square methods. In [7], Uzzal Biswas et.al, used two adaptive filters, such as LMS and normalized LMS are applied to remove the noises. For better clarification simulation results are compared in terms of different performance parameters such as, power spectral density (PSD), spectrogram, frequency spectrum and convergence. Pradeep kumar et.al in [8] presented effective suppression of noise in ECG using wavelet threshodling and empirical mode decomposition methods.

In [11] Dhar et.al, proposed an efficient technique to eliminate high frequency and power line interference noise from digitized Electrocardiogram (ECG) signal. First contaminated ECG signal is passed through a Butterworth low-pass filter to reduce high frequency noises whose order is chosen on experimental basis. To remove power line interference, an improved IIR notch filter is used. Thakor et.al [12] proposed LMS based adaptive recurrent filter for the removal of motion artifacts in ECG signals. Recently Gowri et.al [13-14] variable step size LMS and Dead zone leaky LMS algorithms to reduce noise in the ECG signal.

Normalized LMS and RLS algorithms are used to extract the fetal ECG in [15], they proved that RLS approach gives more noise cancellation than LMS algorithm. Nauman Razzaq et.al [16] used state space RLS algorithm for removing of PLI noise without any reference noise. When input signal is stochastic then Least Mean Square (LMS) algorithm gives good resultant output, but when input signal is deterministic then Recursive Least Square (RLS) algorithm gives better result than LMS algorithm. 


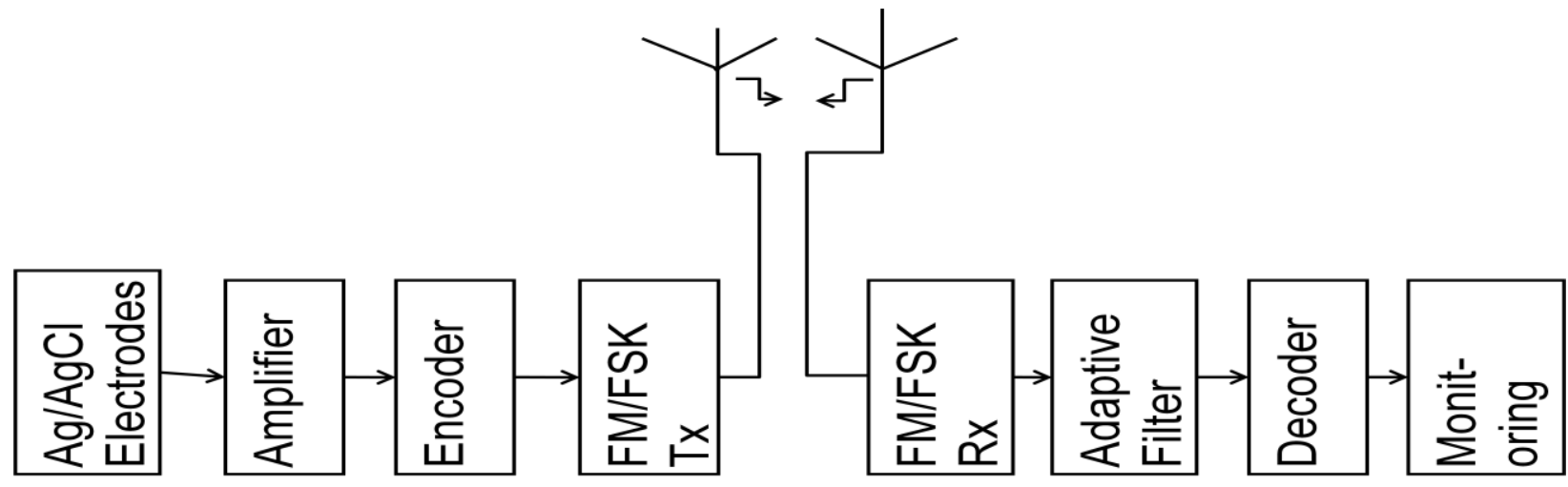

Fig 1: Wireless Ambulatory System

In this paper we proposed efficient posterior RLS algorithm (post-RLS) and for double validation of the signal for better quality, the corrupted signal is passed first LMS algorithm and then passed to RLS algorithm (LMS-RLS). Result analysis shows that post-RLS and LMS-RLS algorithm gives better result compared to RLS and LMS in-terms of Signal to Noise Ratio (SNR) and fast convergence rate.

The rest of the paper is organized as follows. In section 2 , different RLS adaptive filtering technique weight update equations are discussed. In section 3 discussed Simulation results. Finally Section 4 concludes the paper.

\section{EFFICIENT RLS ALGORITHM TO IMPROVE SIGNAL QUALITY}

From few decades onwards adaptive filter play vital role in the elimination of noise in the corrupted signals. A generalized basic adaptive filter structure is shown in Fig 2.

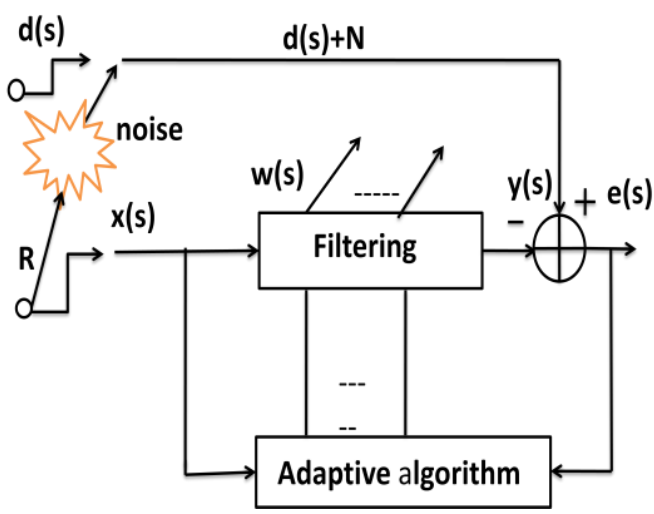

Fig 2: Adaptive filter structure

From the figure the desired signal d(s) along with artifact noise and channel noise is given as primary input. Reference $\mathrm{X}(\mathrm{s})$ input is given to the other input and filter output $\mathrm{y}(\mathrm{s})=\mathrm{X}^{\mathrm{T}}(\mathrm{s}) \mathrm{W}(\mathrm{s}) \quad$ can be obtained by using adaptively adjusted weights according to the output error signal e(s). Weight vector $W(s)=\left[w_{0}(s) w_{1}(s) \ldots w_{L-1}(s)\right]^{T}$ for the length of $\mathrm{L}$, is assumed at initial iteration is zero. If adaptive algorithm is LMS then the weight update equation for this is given as

$\mathrm{W}(\mathrm{s}+1)=\mathrm{W}(\mathrm{s})+\mu \mathrm{e}(\mathrm{s}) \mathrm{X}(\mathrm{s})$,

Where $\mathrm{X}(\mathrm{s})=[\mathrm{x}(\mathrm{s}) \mathrm{x}(\mathrm{s}-1) \ldots \mathrm{x}(\mathrm{s}-\mathrm{L}+1)]$ and output error signal is $e(s)=d(s)-y(s)$. The step size $m u(\mu)$ which controls the error signal, it is in the limit of between 0 and $1 / \lambda \max$, where $\lambda \max$ is maximum Eigen value of the input auto correlation function. Due to depend of this mu factor in LMS algorithm the convergence rate is slow variation in the entire duration of the sampling period. So for improving the convergence rate fast and getting high noise removal from the expected signal we used RLS algorithm. In this RLS algorithm the error signal find out by means of least square method [17], due to this convergence speed increases and in case of LMS algorithm the error signal is find out by means of mean square method due to this also convergence occurs slowly. For the RLS algorithm the cost function for least square error is given by

$$
J(s)=\sum_{j=0}^{s} \lambda^{s-j}\left|e(j)^{2}\right| .
$$

Here $\lambda$ indicates exponential factor, chosen between 0 and 1 , for good noise removal chose $\lambda$ value near to one. The complete recursion of the weight vector is given by

$$
\mathrm{W}(\mathrm{s})=\lambda \mathrm{P}(\mathrm{s}) \mathrm{R}_{\mathrm{dx}}(\mathrm{s}-1)+\mathrm{d}(\mathrm{s}) \mathrm{P}(\mathrm{s}) \mathrm{X}^{*}(\mathrm{~s}) \text {, }
$$

Where $R_{d x}$ is deterministic cross correlation value between desired signal and data input signal. $\mathrm{P}(\mathrm{s})$ is inverse of deterministic cross correlation function. $\mathrm{P}(\mathrm{s})$ is initialized with

$\mathrm{P}(\mathrm{s})=\delta^{-1} \mathrm{I}$, where $\delta$ is chosen small value and $I$, is identity matrix chosen as that of length of order. The simplified priori RLS weight update mathematical equation is given as

$$
\mathrm{W}(\mathrm{s})=\mathrm{W}(\mathrm{s}-1)+\mathrm{e} 1(\mathrm{~s}) \mathrm{g}(\mathrm{s}),
$$

Where e1(s) is priori estimation of the error defined as $\mathrm{e} 1(\mathrm{~s})=\mathrm{d}(\mathrm{s})-\mathrm{W}^{\mathrm{T}}(\mathrm{s}-1) \mathrm{X}(\mathrm{s})$.

Where $\mathrm{g}(\mathrm{s})$ is gain vector, which is given by

$$
g(s)=\frac{R_{x x}^{-1}(s-1) X(s)}{\lambda+X^{T}(s) R_{x x}^{-1}(s-1) X(s)} .
$$

The posterior improved RLS simplified weight equation is given as

$\mathrm{W}(\mathrm{s})=\mathrm{W}(\mathrm{s}-1)+\mathrm{a} e(\mathrm{~s}) \mathrm{g}(\mathrm{s})$ 
Where by randomly chosen 'a' value in between 1 and 2 it gives better result of noise removal compared to RLS, chosen value here is 1.6. The error signal is called posterior error signal given as

$$
\mathrm{e}(\mathrm{s})=\mathrm{d}(\mathrm{s})-\mathrm{W}^{\mathrm{T}}(\mathrm{s}) \mathrm{X}(\mathrm{s}) \text {. }
$$

Comparing with (5) and (8), in equation (8) error signal is calculated after the weight update equation. So comparing with priori RLS with posterior RLS, second one gives fast convergence rate and high signal to noise ratio.

To improve the signal to noise ratio further better and for fast convergence rate, in this paper signal is analyzed with double validation that is, LMS final residue weight vector is taken as initial weight vector for the RLS algorithm. Corrupted ECG signal first passed to the LMS algorithm (1) with initial weight vector length of ' $L$ ' zeros, after complete iteration of the signal final residue weight vector is taken and this weight vector is the initial weight vector for RLS algorithm (4).

Due to this double validation; signal noise can be reduced better in the output signal as compared to above all algorithms. We referred this algorithm as LMS-RLS algorithm. The convergence characteristics for different adaptive algorithms are shown in Fig 3. The LMS to RLS algorithm gives fast convergence rate compared to remaining algorithms with somehow additional complexity. Also, we can observe that the improved posterior RLS algorithm has slightly fast convergence than the RLS algorithm.

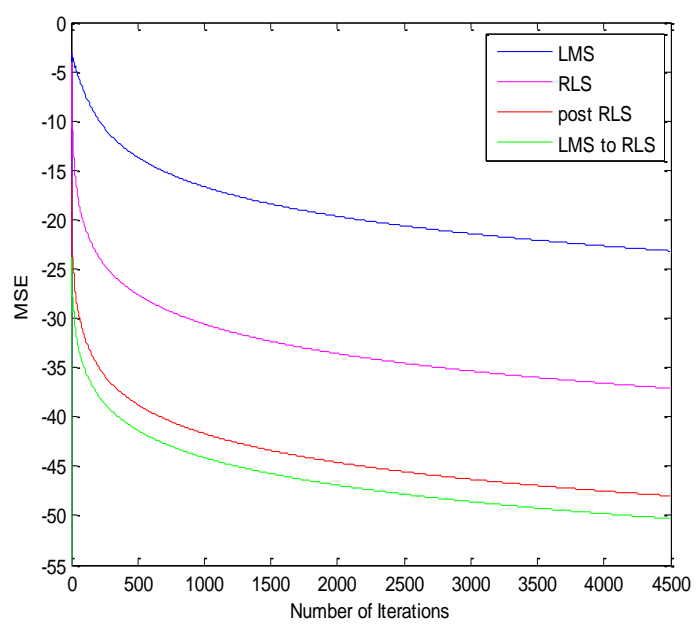

Fig 3: Convergence characteristics for different algorithms

\section{SIMULATION RESULT DISCUSSION}

For analysis of different derived algorithms are best, this adaptive algorithms applied to ECG signal analysis for removing of noise. The pure ECG signal is collected from MIT-BIG physionet database. In this data base totally 47 subjects ECG signal data is available. In this signals some of them are collected from men and some of them are collected from women. These signals are sampled with $360 \mathrm{~Hz}$, with resolution of 11 bit over $10 \mathrm{mV}$ range. From these 47 subjects 15 records collected randomly from this database and added noise and then send to input signal for adaptive filter as shown in Fig 2.
The number of samples collected is 4500. When using measuring instrument for acquiring ECG signal, then power line interference noise is added to automatically to the ECG signal, along with this instrument noise and when transmit signal then channel noise also added. These noises are completely corrupts the original ECG signal, so it should be remove using above mentioned adaptive algorithms. As shown in Fig 4(a) the ECG signal corrupted with PLI and random noise. PLI is sampled with a frequency of $200 \mathrm{~Hz}$. The random noise added here is 0.001 , mu value for LMS algorithm chosen as 0.04 and $\delta$ for RLS algorithm chosen as 0.0001 . From Fig 4(b-e) observe that there is small residue noise in the LMS algorithm, but by using RLS algorithm this noise is almost reduced. In the following figures number of samples taken on $\mathrm{x}$-axis and amplitude is on $\mathrm{y}$-axis.

The performance characteristics are plotted for record number 104. For the performance analysis of different algorithms Signal to Noise Ratio (SNR) calculated for the elimination of PLI noise is shown in Table 1 . From the Table 1 , it is observed that LMS-RLS algorithm gives high reduction of noise compared to remaining algorithms, with SNR of $16.5693 \mathrm{~dB}$ and next place for posterior RLS algorithm gives SNR of $16.3514 \mathrm{~dB}$

The real Muscle artifact noise is collected from MIT-BIH noise stress database. Generally MA noise is high frequency noise compared to other artifacts. This MA noise is added to the pure ECG signal and applied to the adaptive filter. This MA artifact noise added with ECG signal is shown in Fig 5(a). From the Fig 5(b-e) it is observed that, using LMS algorithm there is small MA noise is presented, but using different RLS algorithms the MA noise is better reduced.

As shown in Table 2, various adaptive algorithms for removing of MA noise, from this table analysis, LMS-RLS double validation algorithm gets high SNR of $10.0606 \mathrm{~dB}$, next Posterior RLS gets SNR of $7.6339 \mathrm{~dB}$. For further better analyses these algorithms are applied to remove noise in the recorded speech signal for cross checking. Figure 6(a) is the noisy speech signal and figure 6(b)-6(e) shows the signals after elimination of noises. From these graphs, it can be observed that improved RLS gives high SNR than RLS algorithm and overall noise removal is larger for LMSRLS algorithm. As shown in Table 3, the other performance parameters calculated are Mean Square Error (MSE), Excess MSE (EMSE) and Peak SNR (PSNR) for different adaptive algorithms. From this Table 3, view that LMS-RLS algorithm gets high PSNR (54.4121dB) and low MSE (8.1466e-06) compared to remaining algorithms.

\section{CONCLUSION}

This paper, mainly concentrates to reduce Muscle artifact and Power line interference noise using different RLS based adaptive filters. The different adaptive algorithms used in this paper are LMS, RLS, Posterior RLS and for double validation of the signal we derived LMS-RLS algorithm. For performance analysis of the signal, SNR, MSE, Excess MSE and Peak SNR are calculated. From the above analysis LMSRLS algorithm gives better reduction of noise compared to remaining algorithms with the cost of computational complexity. For somehow reduced complexity the posterior RLS algorithm also gives better output result for elimination of noise. In this paper high reduction of noise is reduced using LMS-RLS algorithm with the cost of computational complexity, for future enhancement of this; the computations may reduce using different adaptive algorithm with better reduction of noise in ECG signal. 


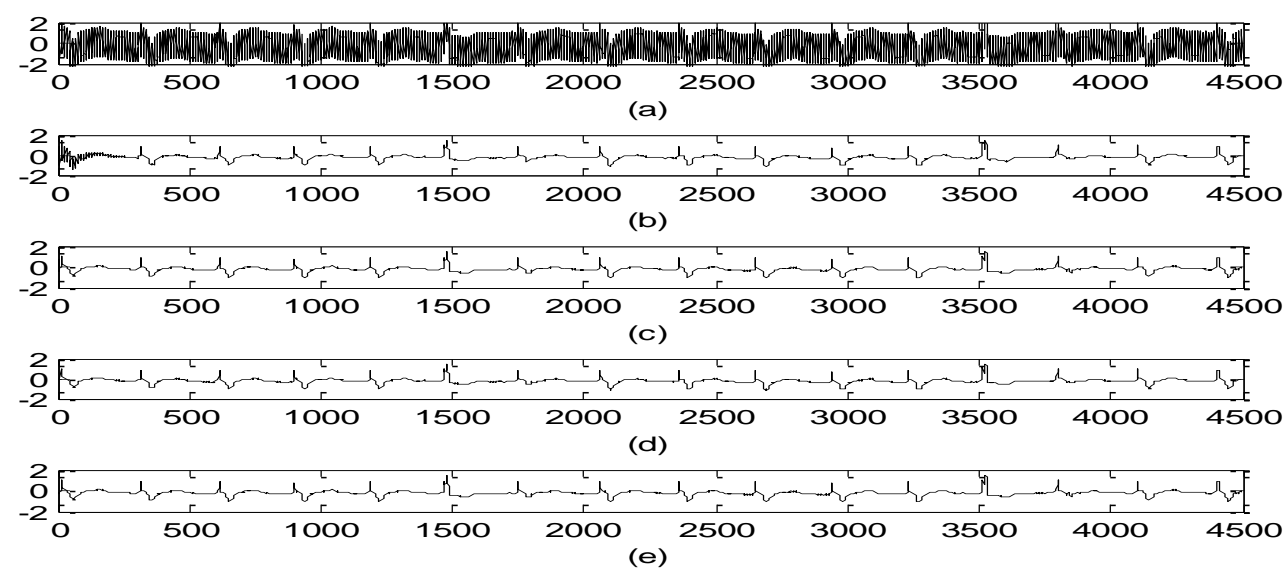

Fig 4: (a) ECG signal corrupted with PLI noise, Noise reduction using (b) LMS algorithm (c) RLS algorithm (d) Posterior RLS and (e) LMS to RLS algorithm.
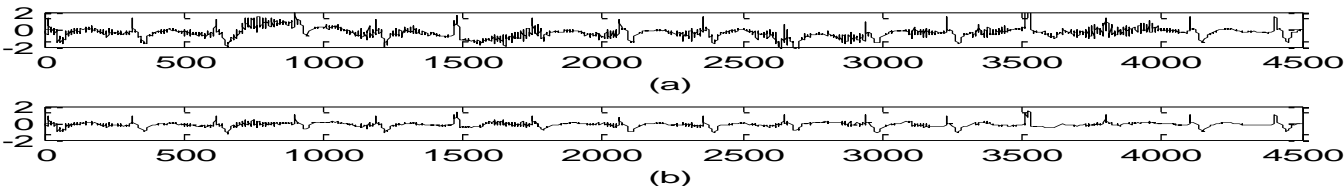

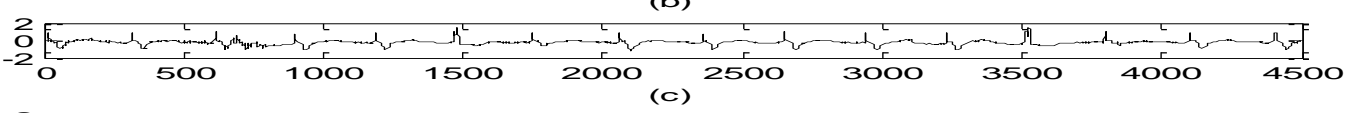

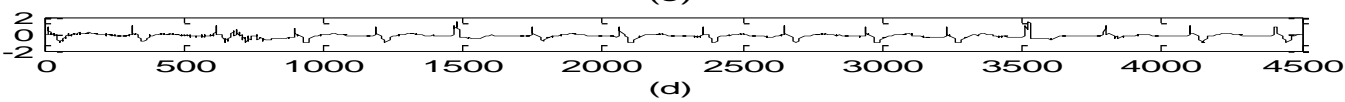

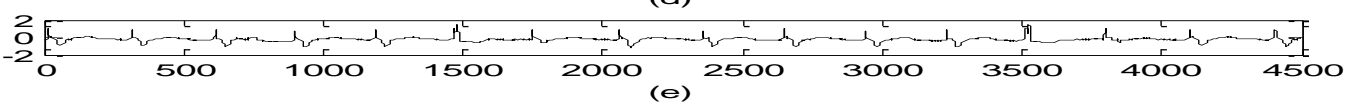

Fig 5: (a) ECG signal corrupted with Muscle artifact noise, Noise reduction using (b) LMS algorithm

(c) RLS algorithm (d) Posterior RLS and (e) LMS to RLS algorithm.

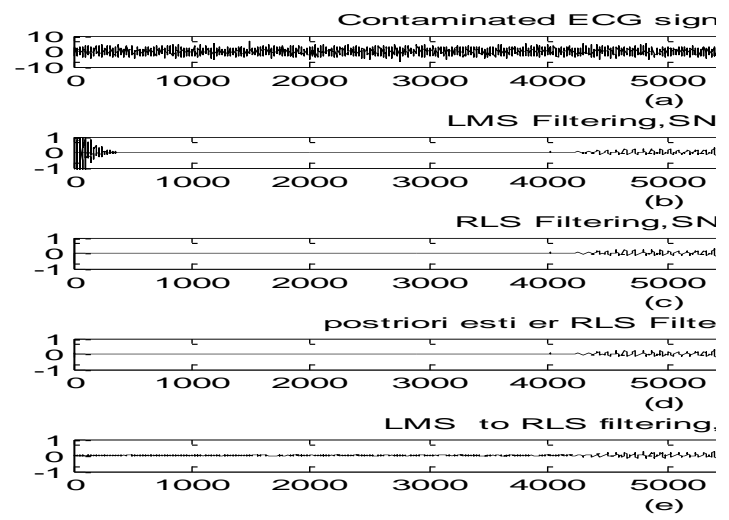

Fig 6: (a) Speech signal corrupted with random noise, Noise reduction using (b) LMS algorithm (c) RLS algorithm (d) Posterior RLS and (e) LMS to RLS algorithm.

Table 1: Performance measure of the different adaptive filters for eliminating of PLI noise in ECG signal.

\begin{tabular}{|c|c|c|c|c|c|c|}
\hline \multirow[b]{2}{*}{ Noise } & \multirow[b]{2}{*}{$\begin{array}{c}\text { Rec } \\
\cdot \\
\text { No. }\end{array}$} & \multirow{2}{*}{$\begin{array}{c}\text { SN } \\
\text { R } \\
\text { Bef } \\
\text { ore } \\
\text { Filte } \\
\text { ring }\end{array}$} & \multicolumn{4}{|c|}{ SNR After Filtering } \\
\hline & & & $\begin{array}{c}\mathrm{LM} \\
\mathrm{S}\end{array}$ & $\begin{array}{c}\text { RL } \\
\text { S }\end{array}$ & $\begin{array}{l}\text { Post- } \\
\text { RLS }\end{array}$ & $\begin{array}{l}\text { LMS- } \\
\text { RLS }\end{array}$ \\
\hline \multirow{4}{*}{ PLI } & 100 & $\begin{array}{l}2.9 \\
263\end{array}$ & $\begin{array}{c}8.79 \\
09\end{array}$ & $\begin{array}{l}14.0 \\
215\end{array}$ & $\begin{array}{c}17.44 \\
84\end{array}$ & $\begin{array}{c}17.62 \\
33\end{array}$ \\
\hline & 102 & $\begin{array}{l}3.8 \\
874\end{array}$ & $\begin{array}{c}7.83 \\
98\end{array}$ & $\begin{array}{c}13.0 \\
06\end{array}$ & $\begin{array}{c}15.79 \\
99\end{array}$ & $\begin{array}{c}16.20 \\
66\end{array}$ \\
\hline & 104 & $\begin{array}{c}- \\
3.1 \\
062\end{array}$ & $\begin{array}{c}8.62 \\
85\end{array}$ & $\begin{array}{l}14.1 \\
022\end{array}$ & $\begin{array}{c}18.74 \\
34\end{array}$ & $\begin{array}{c}19.77 \\
74\end{array}$ \\
\hline & 111 & $\overline{4}$ & 7.36 & 12.4 & 16.08 & 16.15 \\
\hline
\end{tabular}




\begin{tabular}{|c|c|c|c|c|c|}
\hline & 7 & 64 & 53 & 03 & 41 \\
\hline 114 & $\begin{array}{c}- \\
5.2 \\
854\end{array}$ & $\begin{array}{c}6.47 \\
83\end{array}$ & $\begin{array}{l}11.6 \\
619\end{array}$ & $\begin{array}{c}15.81 \\
38\end{array}$ & $\begin{array}{c}15.93 \\
31\end{array}$ \\
\hline 202 & $\begin{array}{c}- \\
4.7 \\
304\end{array}$ & $\begin{array}{c}6.99 \\
93\end{array}$ & $\begin{array}{l}11.9 \\
064\end{array}$ & $\begin{array}{c}15.06 \\
74\end{array}$ & $\begin{array}{c}15.12 \\
85\end{array}$ \\
\hline 207 & $\begin{array}{c}- \\
2.9 \\
976\end{array}$ & $\begin{array}{c}8.68 \\
81\end{array}$ & $\begin{array}{l}13.6 \\
314\end{array}$ & $\begin{array}{c}15.95 \\
16\end{array}$ & $\begin{array}{c}16.12 \\
45\end{array}$ \\
\hline 208 & $\begin{array}{c}1.1 \\
358\end{array}$ & $\begin{array}{c}10.2 \\
58\end{array}$ & $\begin{array}{l}13.8 \\
652\end{array}$ & $\begin{array}{c}14.74 \\
39\end{array}$ & $\begin{array}{c}14.77 \\
79\end{array}$ \\
\hline 209 & $\begin{array}{c}- \\
4.1 \\
455\end{array}$ & $\begin{array}{c}7.54 \\
44\end{array}$ & $\begin{array}{l}12.2 \\
165\end{array}$ & $\begin{array}{c}14.58 \\
24\end{array}$ & $\begin{array}{c}14.61 \\
67\end{array}$ \\
\hline 210 & $\begin{array}{c}- \\
3.9 \\
608\end{array}$ & $\begin{array}{c}7.77 \\
73\end{array}$ & $\begin{array}{l}12.9 \\
942\end{array}$ & $\begin{array}{c}17.05 \\
97\end{array}$ & $\begin{array}{c}17.16 \\
76\end{array}$ \\
\hline 214 & $\begin{array}{c}1.6 \\
192\end{array}$ & $\begin{array}{c}10.0 \\
47\end{array}$ & $\begin{array}{l}15.2 \\
547\end{array}$ & $\begin{array}{c}18.70 \\
26\end{array}$ & $\begin{array}{c}18.89 \\
04\end{array}$ \\
\hline 222 & $\begin{array}{l}5.9 \\
639\end{array}$ & $\begin{array}{c}5.81 \\
41\end{array}$ & $\begin{array}{l}11.3 \\
304\end{array}$ & $\begin{array}{c}17.46 \\
24\end{array}$ & $\begin{array}{c}17.81 \\
69\end{array}$ \\
\hline 228 & $\begin{array}{l}3.6 \\
45\end{array}$ & $\begin{array}{c}8.04 \\
39\end{array}$ & $\begin{array}{l}12.9 \\
201\end{array}$ & $\begin{array}{c}15.27 \\
25\end{array}$ & $\begin{array}{c}15.39 \\
44\end{array}$ \\
\hline 233 & $\begin{array}{c}- \\
0.5 \\
181\end{array}$ & $\begin{array}{c}10.9 \\
75\end{array}$ & $\begin{array}{l}15.3 \\
004\end{array}$ & $\begin{array}{c}16.53 \\
62\end{array}$ & $\begin{array}{c}16.88 \\
86\end{array}$ \\
\hline 234 & $\begin{array}{c}- \\
3.0 \\
692\end{array}$ & $\begin{array}{c}8.61 \\
16\end{array}$ & $\begin{array}{c}13.4 \\
66\end{array}$ & $\begin{array}{c}16.00 \\
68\end{array}$ & $\begin{array}{c}16.03 \\
99\end{array}$ \\
\hline \multicolumn{2}{|c|}{ Average } & $\begin{array}{c}8.25 \\
75\end{array}$ & $\begin{array}{r}13.2 \\
086\end{array}$ & $\begin{array}{c}16.35 \\
14\end{array}$ & $\begin{array}{c}16.56 \\
93\end{array}$ \\
\hline
\end{tabular}

Table 2: Performance measure of the different adaptive filters for eliminating of MA noise in ECG signal

\begin{tabular}{|c|c|c|c|c|c|}
\hline \multirow{4}{*}{ Noise } & \multirow{2}{*}{$\begin{array}{c}\text { Rec. } \\
\text { No. }\end{array}$} & \multicolumn{4}{|c|}{ SNR after filtering } \\
\cline { 3 - 6 } & LMS & RLS & $\begin{array}{c}\text { Post- } \\
\text { RLS }\end{array}$ & $\begin{array}{c}\text { LMS- } \\
\text { RLS }\end{array}$ \\
\hline \multirow{7}{*}{} & 100 & 3.8171 & 5.4971 & 5.6844 & 11.5618 \\
\cline { 2 - 6 } & 102 & 4.341 & 6.4641 & 6.9149 & 13.0312 \\
\cline { 2 - 6 } & 104 & 4.5039 & 7.41 & 7.5176 & 14.156 \\
\cline { 2 - 6 } & 111 & 4.9041 & 7.6491 & 7.7184 & 12.1946 \\
\cline { 2 - 6 } & 114 & 3.8085 & 5.2855 & 5.566 & 7.9896 \\
\cline { 2 - 6 } & 202 & 5.0316 & 7.9039 & 8.1363 & 4.9579 \\
\cline { 2 - 6 } & 207 & 3.3087 & 6.5924 & 6.7399 & 6.3964 \\
\cline { 2 - 6 } & 208 & 5.1171 & 8.234 & 8.4956 & 13.9413 \\
\cline { 2 - 6 } & 209 & 4.9295 & 8.0095 & 8.1228 & 13.5261 \\
\cline { 2 - 6 } & 210 & 5.4417 & 7.7423 & 7.8068 & 12.0156 \\
\cline { 2 - 6 } & 214 & 5.2143 & 9.9076 & 10.1657 & 11.3899 \\
\cline { 2 - 6 } & 222 & 4.6927 & 6.2755 & 6.4342 & 6.8678 \\
\hline
\end{tabular}

\begin{tabular}{|l|c|c|c|c|c|}
\hline \multirow{7}{*}{228} & 4.1109 & 5.4246 & 5.5977 & 8.3105 \\
\cline { 2 - 6 } & 233 & 4.6907 & 9.1423 & 9.4519 & 9.1617 \\
\cline { 2 - 6 } & 234 & 5.0585 & 10.035 & 10.1566 & 5.4099 \\
\cline { 2 - 6 } Avg & $\mathbf{4 . 5 9 8 0}$ & $\mathbf{7 . 4 3 8 2}$ & $\mathbf{7 . 6 3 3 9}$ & $\mathbf{1 0 . 0 6 0 6}$ \\
\hline
\end{tabular}

Table 3: MSE, Excess MSE, and Peak SNR of different adaptive filters, for eliminating of PLI noise in ECG signal

\begin{tabular}{|c|c|c|c|c|}
\hline $\begin{array}{c}\text { ALGORI } \\
\text { THM. }\end{array}$ & $\begin{array}{c}\text { Rec. } \\
\text { No. }\end{array}$ & MSE & $\begin{array}{c}\text { EMSEss } \\
(\mathrm{dB})\end{array}$ & $\begin{array}{c}\text { PSNR } \\
(\mathrm{dB})\end{array}$ \\
\hline \multirow{2}{*}{ LMS } & 102 & 0.0022 & -44.4963 & 26.1254 \\
\cline { 2 - 5 } & 104 & 0.0022 & -42.8362 & 30.0090 \\
\hline \multirow{2}{*}{ RLS } & 102 & $\begin{array}{c}1.7613 \mathrm{e}- \\
04\end{array}$ & -66.1370 & 37.1418 \\
\cline { 2 - 5 } & 104 & $\begin{array}{c}1.7579 \mathrm{e}- \\
04\end{array}$ & -65.4815 & 41.0720 \\
\hline \multirow{2}{*}{$\begin{array}{c}\text { POSTRIER } \\
\text { RLS }\end{array}$} & 102 & $\begin{array}{c}1.9485 \mathrm{e}- \\
05\end{array}$ & -61.0597 & 46.7031 \\
\cline { 2 - 5 } & 104 & $\begin{array}{c}1.4339 \mathrm{e}- \\
05\end{array}$ & -60.8412 & 51.9567 \\
\hline \multirow{2}{*}{$\begin{array}{c}\text { LMS TO } \\
\text { RLS }\end{array}$} & 102 & $\begin{array}{c}1.4665 \mathrm{e}- \\
05\end{array}$ & -61.1651 & 47.9371 \\
\cline { 2 - 5 } & 104 & $\begin{array}{c}8.1466 \mathrm{e}- \\
06\end{array}$ & -63.1446 & 54.4121 \\
\hline
\end{tabular}

\section{REFERENCES}

[1] Kestler HA, Haschka M, Kratz W, Schwenker F, Palm G, Hombach V, Hoher M. 1998 "De-noising of highresolution ECG signals by combining the discrete wavelet transform with the Wiener filter". Comput. Cardiol. 25:pp.233-236.

[2] James C. Huhta, John G. Webster, 1973 “60-Hz Interference in Electrocardiography", IEEE Transactions on Biomedical Engineering, vol. BME-20, no. 2, pp. 91-101.

[3] A Alesanco and J Garc'1a, 2010 "Clinical Assessment of Wireless ECG Transmission in Real-Time Cardiac Telemonitoring", IEEE Transactions on Information Technology in Biomedicine, vol. 14, no. 5, pp. 11441152.

[4] Chin-Teng Lin, Kuan-Cheng Chang, Chun-Ling Lin, Chia-Cheng Chiang, Shao-Wei Lu, Shih-Sheng Chang, Bor-Shyh Lin, Hsin-Yueh Liang, Ray-Jade Chen, YuanTeh Lee, and Li-Wei Ko, 2010 "An Intelligent Telecardiology System using a Wearable and Wireless ECG to Detect Atrial Fibrillation", IEEE Transactions on Information Technology in Biomedicine, vol. 14, no. 3, pp.726-733.

[5] SangJoon Lee, Jungkuk Kim and Myoungho Lee, 2011 "A Real-Time ECG Data Compression and Transmission Algorithm for an e-Health Device", IEEE Transactions On Biomedical Engineering, vol. 58, no. 9, pp.24482454.

[6] Yunfeng Wu, Rangaraj M. Rangayyan, Yachao Zhou and Sin-Chun Ng,2009 "Filtering electrocardiographic signals using an unbiased and normalized adaptive noise reduction system", Medical Engineering \& Physics, Elsevier, vol. 31, pp. 17-26.

[7] Biswas, U.; Das, A.; Debnath, S.; Oishee, I.,2014 "ECG signal denoising by using least-mean-square and normalised-least-mean-square algorithm based adaptive 
filter," in Informatics, Electronics \& Vision (ICIEV), International Conference on , vol., no., pp.1-6, 23-24.

[8] Pradeep Kumar, B.; Balambigai, S.; Asokan, R., 2012 "ECG de-noising based on hybrid technique," in Advances in Engineering, Science and Management (ICAESM), International Conference on , pp.285-290.

[9] Rik Vullings, Bert de Vries, and Jan W. M. Bergmans, 2011 "An Adaptive Kalman Filter for ECG Signal Enhancement", IEEE Transactions on Biomedical Engineering, vol. 58, no. 4, pp.1094-1103.

[10] Bhavani Sankar, A., D. Kumar, K. Seethalakshmi, 2012 "A New Self-Adaptive Neuro Fuzzy Inference System for the Removal of Non-Linear Artifacts from the Respiratory Signal”. Journal of Computer Science 8 (5): 621-631.

[11] Dhar, S.; Mukhopadhyay, S.K.; Mitra, S.; Baig, M.M.; Mitra, M., 2014 "Noise reduction and lossless ECG encoding," in Control, Instrumentation, Energy and Communication (CIEC), International Conference on , vol., no., pp.210-213

[12] N. V. Thakor and Yi-Sheng Zhu, 1991 "Applications of adaptive filtering to ECG analysis: noise cancellation and arrhythmia detection", IEEE Transactions on Biomedical Engineering, vol. 38, no. 8, pp.785-794.

[13] Gowri T, Rajesh Kumar P, Rama Koti Reddy DV. 2014 "An Efficient Variable Step Size Least Mean Square
Adaptive Algorithm Used to Enhance the Quality of Electrocardiogram Signal", Advances in intelligent systems and computing; 264; PP.463-475.

[14] Gowri T, Rajesh Kumar P, Rama Koti Reddy DV, Rehaman Md, 2015 "Removal of Artifacts from Electrocardiogram Using Efficient Dead Zone Leaky LMS Adaptive Algorithm"Proceedings of 3rd International Conference on Advanced Computing, Networking and Informatics, Smart Innovation, Systems and Technologies 43, PP.619-630.

[15] Shi-jin Liu, Da-Ii Liu, Jing-quan Zhang, Yan-jun Zeng, 2011 "Extraction of Fetal Electrocardiogram using Recursive Least Squares and Normalized Least Mean Squares Algorithms". International Conference on Advanced Computer Control pp.333-336.

[16] Nauman Razzaq, Maryam Butt, Muhammad Salman, Rahat Ali, Ismail Sadiq, Khalid Munawar, Tahir Zaidi, 2013, "Self Tuned SSRLS Filter for Online Tracking and Removal of Power Line Interference from Electrocardiogram", Proceedings of International Conference on Modelling, Identification \& Control, pp.339-343.

[17] Haykin, S., 1986 Adaptive Filter Theory, Eaglewood Clirs, NJ: Prentice-Hall 